\title{
BMJ A retrospective cohort study: open 10-year trend of disease-modifying antirheumatic drugs and biological agents use in patients with rheumatoid arthritis at Veteran Affairs Medical Centers
}

\author{
Bernard Ng, ${ }^{1,2}$ Adeline Chu, ${ }^{3}$ Myrna M Khan ${ }^{1,2}$
}

To cite: $\mathrm{Ng} \mathrm{B}$, Chu A, Khan MM. A retrospective cohort study: 10-year trend of disease-modifying antirheumatic drugs and biological agents use in patients with rheumatoid arthritis at Veteran Affairs Medical Centers. BMJ Open 2013;3:e002468.

doi:10.1136/bmjopen-2012002468

- Prepublication history and additional material for this paper are available online. To view these files please visit the journal online (http://dx.doi.org/10.1136/ bmjopen-2012-002468).

Received 7 December 2012 Revised 11 March 2013 Accepted 12 March 2013

This final article is available for use under the terms of the Creative Commons Attribution Non-Commercial 2.0 Licence; see http://bmjopen.bmj.com

For numbered affiliations see end of article.

Correspondence to Dr Bernard Ng; bernardng1@gmail.com

\section{ABSTRACT}

Objectives: To evaluate the trends in patterns of disease-modifying antirheumatic drugs (DMARDs) and biological agents use from 1999 to 2009 and to identify patient characteristics associated with different patterns of their use in a national sample of Veterans with rheumatoid arthritis (RA).

Design: A retrospective cohort study.

Settings: Administrative databases of the USA Department of Veterans Affairs.

Participants: An incident cohort of 13254 patients with newly diagnosed RA was identified.

Primary outcome measures: Trends and choice of DMARDs and biological agents' usage, and time intervals between RA diagnosis and treatment

Results: Methotrexate use as first-line agent increased from $39.9 \%$ to $57.2 \%$ over the study period $(p<0.001)$. Although biological dispensations increased over other DMARDs and biological agents, from $3.4 \%$ to $25 \%$ from 1999 to 2009, the percentage of RA patients diagnosed between 1999 and 2007 who had biologics dispensations remained steady at $23.3-26.7 \%$. Compared with Caucasian, African Americans were less likely to receive biologics (HR $0.71,95 \% \mathrm{Cl} 0.63$ to 0.81 ). Patients aged 75 and older were less likely to receive biologics than those younger than 45 (HR 0.29, $95 \% \mathrm{Cl} 0.23$ to 0.36 ). The time interval between RA diagnosis and treatment with DMARDs and biological agents decreased significantly over time (median: 51 days in 1999-2001 to 28 days in 2006-2007). Conclusions: Methotrexate use increased as it became the preferred first-line agent, while other traditional agents declined. Dispensation of biologics increased significantly, but the proportion of RA patients eventually given biologics stabilised below $30 \%$. A significant shorter time between RA diagnosis and DMARD or biological agent initiation in recent years suggests improvements in quality of care. There were disproportionately lower use of biologics in certain age and ethnic groups, and further studies will be needed to elucidate these observations.

\section{ARTICLE SUMMARY}

Article focus

- There are significant changes in patterns of antirheumatic agents used since the introduction of biological agents.

- Patient factors may affect the choice of antirheumatic agents.

- The use of various antirheumatic agents may be in different patient groups: for example, biological agents use may be higher in certain ethnic groups.

Key messages

- Using national database to study the changes in prescribing trends of antirheumatic agents in a large incident cohort of patients with rheumatoid arthritis and following them across a 10-year time period.

- Able to study the changes in the duration of use of various antirheumatic agents over time which is not possible with cross-sectional studies.

- Assess the changes in quality of care over the years for RA patients using surrogates such as time between RA diagnosis to initiation of antirheumatic agents.

Strengths and limitations of this study

- Large cohort size and long follow-up period. No recall biases.

- Data on women and minority groups were limited, but are reflective of the Veterans population.

\section{INTRODUCTION}

Clinical trials have established the efficacy and low risk-to-benefit ratio of methotrexate (MTX) in the treatment of rheumatoid arthritis (RA) since the early 1980 s. $^{1}{ }^{2}$ The concerns over adverse events slowed acceptance of MTX over hydroxychloroquine (HCQ) 
and sulfasalazine (SSZ), and in the early 1990s, MTX was still considered as a second-line agent for RA treatment. ${ }^{3}$ It was not until a decade later that MTX was accepted as the main drug for treating RA, showing the superiority of MTX over HCQ and SSZ. ${ }^{4}$

In the late 1990s, the range of pharmacotherapy available for RA had greatly expanded with the introduction of biologics. ${ }^{5}$ Studies showed that physicians' preference played a more important role than the patients' disease activity when choosing biologics over disease-modifying antirheumatic drugs (DMARDs) and that biologics were being used in patients with less severe disease over time. ${ }^{6} 7$ The use of biologics has not been uniform in the USA. A Medicaid database study suggested that African Americans had lower odds of receiving biologics for RA treatment compared with Caucasians. ${ }^{8}$

This study examined (1) trends of DMARDs and biological agents use, (2) choice of DMARDs and biological agents and (3) time intervals between RA diagnosis and treatment over the past 10 years in the US Department of Veteran Affairs (VA) healthcare system.

\section{PATIENTS AND METHODS}

The study was approved by the Baylor College of Medicine Institutional Review Board and the Michael E DeBakey VA Medical Center Research and Development Committee.

\section{Study population and data sources}

The research was carried out with the National Veterans Health Administration administrative databases: VA inpatient files, VA outpatient clinic files, vital status files, pharmacy benefits management files and decision support system laboratory files. These databases provided a comprehensive documentation of all Veterans across the nation. A person who has served in the active military service in the USA and who was discharged honourably from service is considered a Veteran and would be eligible to access VA healthcare. Using these databases, we identified a cohort of RA patients receiving healthcare at the VA between 1 October 1999 and 30 September 2009 using the following criteria: (1) two or more RA diagnosis codes (International Classification of Diseases 9 (ICD9) code 714) at least 6 months apart; (2) at least one rheumatology clinic visit; (3) RA diagnosis code entered at the last rheumatology clinic visit and (4) at least one DMARD or biological agent dispensed for a total duration of 6 months or more. The following DMARDs and biological agents were considered: MTX, Azathioprine, Leflunomide, SSZ, HCQ, Gold and Minocycline (DMARDs); Adalimumab, Etanercept, Infliximab, Golimumab, Certolizumab, Abatacept, Anakinra, Rituximab and Tocilizumab (biological agents). In a recent study, chart review was done in a random sample of patients with RA diagnosis code to ascertain the accuracy of various algorithms, and the specificity and positive predictive value of above algorithm for the database diagnosis of RA were $98.4 \%$ and $91.4 \%$, respectively. ${ }^{9}{ }^{10}$ One of the criteria used in our chart review for establishing diagnosis of RA was the ACR 1987 criteria. It is not possible to use ACR criteria to diagnose RA in administrative databases because ACR criteria for RA are not typically captured in such databases.

To define incident RA cohort, we added the following criteria: (1) be in the VA health system for at least 2 years before the first RA diagnosis code, and (2) had at least two encounters within those 2 years without an RA diagnosis code and were not dispensed any of the above DMARDs and biological agents. This screening process involved searching the databases back to 1 October 1997.

\section{DMARDs and biological agents use from 1999 to 2009}

The study period between 1 October 1999 and 30 September 2009 was grouped into five time periods to reduce the variability between individual years. We examined DMARDs and biological agents that were dispensed continuously for a total of 90 days or more. Dispensations with gaps that were less than 14 days were considered as continuous. For any particular DMARD or biological agent, we used date of first release date as the start date of the first continuous 90-day dispensation. This analysis is independent of the 180 days or more criterion that was used to define the RA cohort. For the analysis of first DMARD or biological agent usage, if there were two or more drugs started on the same date, we captured all as the first DMARD or biological agent used. Therapeutic change was defined as the switch to or addition of a second DMARD or biological agent.

\section{Triple therapy with MTX, HCQ and SSZ}

The start date of triple therapy was defined as the date when the third DMARD (MTX, HCQ or SSZ) was dispensed and the first two DMARDs were still active. There must be at least 30 days of overlap for all three DMARDs.

\section{Statistical analyses}

We evaluated the characteristics of the study cohort using data of patients diagnosed with RA in each of the five time periods (1999-2001, 2002-2003, 2004-2005, 2006-2007 and 2008-2009). We included demographic and clinical variables such as comorbidity scores and laboratory test results. Comorbidity scores were adapted from the Deyo-Charlson comorbidity index. ICD9 codes related to RA, such as 714.xx and 725, were excluded to avoid bias. ${ }^{11-13}$ The $\chi^{2}$ tests for homogeneity were used for categorical variables (sex, age categories, ethnicity and comorbidity scores). Linear regression models were used for continuous variables, such as laboratory results. Trends of DMARDs and biological agents used across the five time periods were compared by CochraneArmitage trend tests. 
Multivariable logistic regression models were used to compare the factors that may influence the choice of one DMARD or biological agent as the first-line agent over another. The logistic regression model was adjusted for specific baseline data: age categories at RA diagnosis, sex, ethnicity, comorbidity scores, serum $\mathrm{C}$ reactive protein (CRP), serum creatine, haemoglobin, white blood cell count, serum alanine aminotransferase (ALT) and serum aspartate aminotransferase.

Cox regression models were used to analyse the associations between the interval from RA diagnosis to the various events and exact year the patients were diagnosed with RA. The events were (1) start of first DMARD; (2) start of biologics and (3) therapeutic change. The RA diagnostic criterion that required the use of at least one DMARD or biological agent resulted in under-representation of subjects with long intervals between RA diagnosis and start of the first agent when RA diagnosis was near the end of the study. Therefore, in the model analysing time intervals between RA diagnosis and the start of the first DMARD or biological agent, we only included patients who had RA diagnosed between 1 October 1999 and 30 September 2007 and had their first DMARD or biological agent started within 720 days from the date of RA diagnosis to avoid systematic errors. The period of 720 days was chosen to allow for sufficient time between the cut-off date of RA diagnosis (30 September 2007) and the end of follow-up period (30 September 2009). We did not apply time restrictions in the following two Cox regression models because the events were not similarly affected by the definition our RA cohort. In the therapeutic change model, patients who started combination therapy as their first antirheumatic therapy were excluded. All three Cox regression models were adjusted for serum CRP and specific baseline data which had significant variations over time. The percentage difference in the HR of an event between time periods were calculated by using the formula: $(\mathrm{HR} /(\mathrm{HR}+1)) \times 100$.

All statistical analyses were carried out with STATA V.12.0, College Station, Texas, USA, and SAS statistical software V.9.2, Cary, North Carolina, USA.

\section{RESULTS}

A total of 13254 patients met our RA incident cohort criteria. There was a 9:1 male-to-female preponderance which was expected in a VA population. Age at diagnosis had significant variations across the time periods. Declines in percentage over time were most notable in the two younger age categories: $<45$ and $45-54$ $(p<0.001)$. There were also significant changes in the ethnicity distribution across the five time periods with gradual declines in percentages of African Americans and others (Hispanics/Asians) ethnic group categories $(\mathrm{p}<0.001$; table 1$)$.

A majority of DMARD-naïve patients were started on a single DMARD or biological agent $(92.7 \%)$. The most frequent combination therapy used was with two DMARDs $(76.1 \%)$. Less than $2 \%$ were started on triple DMARDs. MTX was the most commonly used first DMARD alone or in combination with other DMARDs and biological agents. Its use as the first DMARD or biological agent increased from 39.9\% in 1999-2001 to $57.2 \%$ in $2008-2009(\mathrm{p}<0.001)$. The use of biologics as the first DMARD or biological agent also increased significantly from $3 \%$ in $1999-2001$ to $6.7 \%$ in 2006-2007 $(\mathrm{p}<0.001)$. The use of SSZ as the first agent for RA treatment declined significantly from $16 \%$ in 1999-2001 to 9.6\% in 2008-2009 $(\mathrm{p}<0.001)$. Use of HCQ as the first RA treatment agent also declined, but to a lesser extent over the same periods: from $37 \%$ to $33.7 \% \quad(p=0.04)$. The use of Leflunomide as the first DMARD or biological agent remained stable between $2.8 \%$ to $3.6 \%$ ( $\mathrm{p}=0.65$; Table 2).

The results from our analyses on each patient for all DMARDs and biological agents used were similar to the first DMARD or biological agent usage results. The number of patients who had ever been dispensed MTX increased from $61.4 \%$ in $1999-2001$ to $69.7 \%$ in 2008 $2009(\mathrm{p}<0.001)$, while those who had been dispensed HCQ or SSZ declined significantly over the same time periods (both $\mathrm{p}<0.001$; see table 2 ). Leflunomide use also trended lower $(\mathrm{p}<0.001)$. An interesting finding was that the percentage of RA patients using biologics over the first 4 time periods remained relatively stable $(p=0.67)$. In view of the significantly longer time interval between RA diagnosis and the start of first biological when compared with the start of the first DMARD or biological agent (median interval: 1576 vs 39 days, $\mathrm{p}<0.001$ ), and our follow-up period ending on 30 September 2009, patients diagnosed in 2008-2009 have insufficient follow-up time to observe their switch to biologics, resulting in an apparent lower percentage of biological use for this time period. We also found that the number of biologics dispensations increased from $3.4 \%$ of all DMARDs and biological agents dispensed in 19992001 to $25 \%$ in $2008-2009$.

Logistic regression showed the increased likelihood of MTX use as the first DMARD over HCQ and SSZ in the more recent years (table 3 ). We corrected specific baseline data as mentioned in the methods section. In the same model, male patients were associated with the increased odds of MTX use compared with HCQ (OR 1.58). The opposite was true when MTX was compared with SSZ: men had lower odds of MTX use (OR 0.72). When comparing patients aged 75 and older to those less than 45, MTX was more likely to be used than HCQ and SSZ, ORs 1.59 and 3.07, respectively. There was more use of MTX over SSZ among Hispanics and Asians when compared with Caucasians (OR 1.50).

The median time interval between RA diagnosis and the start of the first DMARD or biological agent decreased from 51 days (IQR:227) in 1999-2001 to 28 days in 2006-2007 (IQR:71; median ratio (MR) 1.82). In the Cox regression model, with 1999-2001 as 
Table 1 Baseline data: demographics, modified comorbidity score and laboratory results

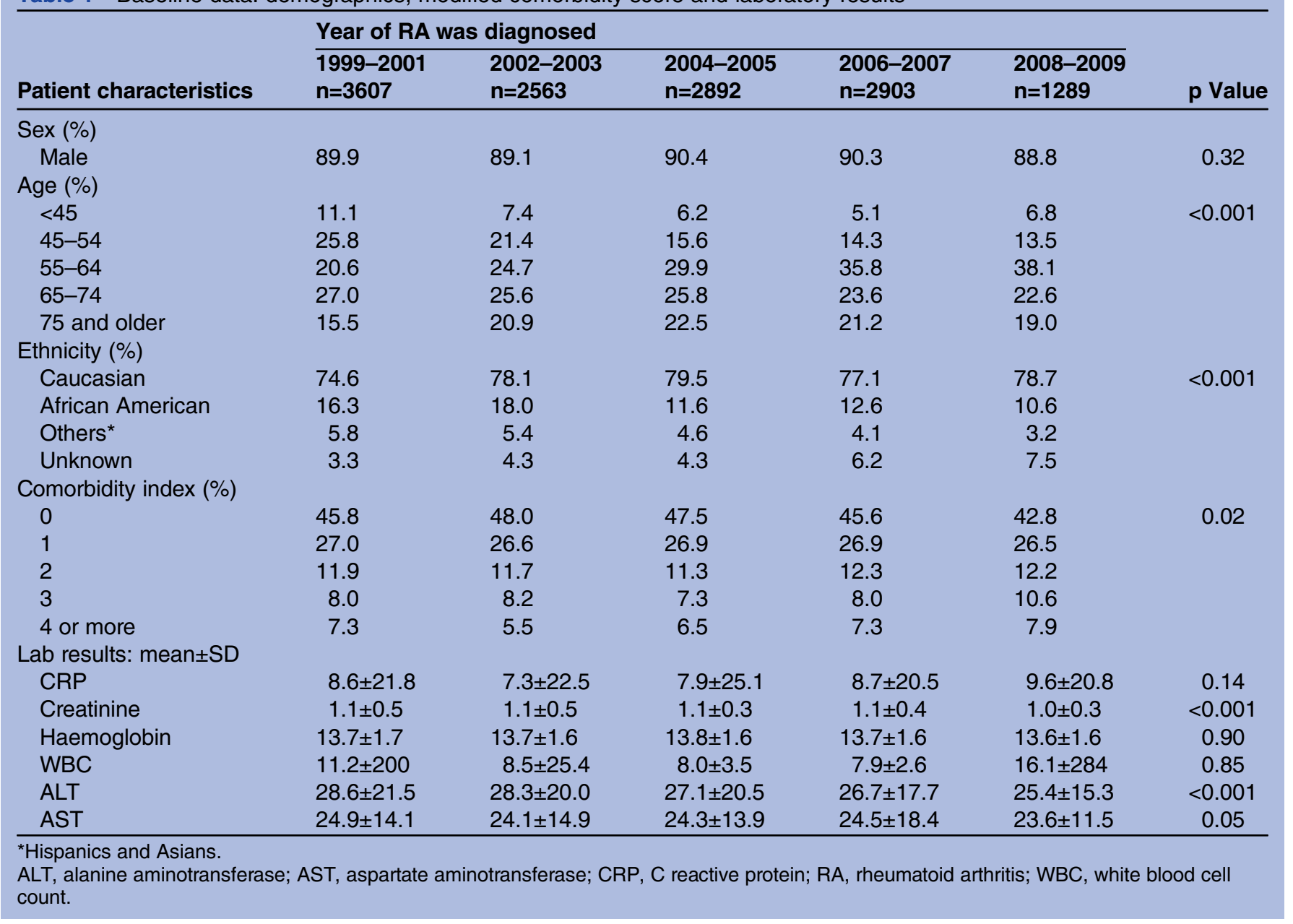

reference, the HR for the start of the treatment was 1.33 for those diagnosed with RA in 2006-2007. Significantly higher odds of starting the first DMARD or biological agent earlier were observed in older age categories ( $\mathrm{p}<0.001$; table 4$)$. Adjustments were made for ethnicity, comorbidity scores, serum CRP, serum creatine and serum ALT.
The median time between RA diagnosis and the start of the first biological agent was 3227 days (IQR 705) in 1999-2001 and 414 days (IQR 232) in 2008-2009 (MR 7.79). Those diagnosed with RA in 2008-2009 had a $74 \%$ higher chance of an earlier start on biologics than those diagnosed in 1999-2001 (table 4). The rate at which the biologics were initiated increased across the

Table 2 Percentage rheumatoid arthritis patients started on antirheumatic agents as their first agent or as given in any sequence

\begin{tabular}{|c|c|c|c|c|c|c|c|c|c|c|c|c|}
\hline \multirow[b]{3}{*}{ Antirheumatic agents } & \multicolumn{10}{|c|}{ Year of RA was diagnosed } & \multirow{2}{*}{\multicolumn{2}{|c|}{$\begin{array}{l}\text { Trend of odds } \\
\text { p Value }\end{array}$}} \\
\hline & \multicolumn{2}{|c|}{$\begin{array}{l}\frac{1999-2001}{n=3607} \\
\end{array}$} & \multicolumn{2}{|c|}{$\begin{array}{l}\frac{2002-2003}{n=2563} \\
\end{array}$} & \multicolumn{2}{|c|}{$\frac{2004-2005}{n=2892}$} & \multicolumn{2}{|c|}{$\begin{array}{l}\frac{2006-2007}{n=2903} \\
\end{array}$} & \multicolumn{2}{|c|}{$\begin{array}{l}\frac{2008-2009}{n=1289} \\
\end{array}$} & & \\
\hline & $\overline{\text { First }}$ & Any & $\overline{\text { First }}$ & $\overline{\text { Any }}$ & $\overline{\text { First }}$ & $\overline{\text { Any }}$ & $\overline{\text { First }}$ & $\overline{\text { Any }}$ & $\overline{\text { First }}$ & Any & $\overline{\text { First }}$ & Any \\
\hline Methotrexate (\%) & 39.9 & 61.4 & 45.9 & 64.9 & 51.2 & 67.9 & 51.8 & 66.9 & 57.2 & 69.7 & $<0.001$ & $<0.001$ \\
\hline loroquine (\%) & 37.0 & 54.3 & 36.5 & 54.9 & 35.3 & 53.5 & 35.8 & 51.4 & 33.7 & 46.5 & 0.04 & $<0.001$ \\
\hline Sulfasalazine (\%) & 16.0 & 33.7 & 12.8 & 28.8 & 10.9 & 25.5 & 8.8 & 20.6 & 9.6 & 19.4 & $<0.001$ & $<0.001$ \\
\hline Leflunomide (\%) & 3.0 & 16.9 & 3.4 & 16.0 & 2.8 & 15.6 & 3.6 & 11.6 & 2.9 & 7.9 & 0.65 & $<0.001$ \\
\hline Other traditional DMARDs (\%) & 5.9 & 14.7 & 4.1 & 10.7 & 3.0 & 7.6 & 2.9 & 6.2 & 1.3 & 3.6 & $<0.001$ & $<0.001$ \\
\hline Biologics (\%) & 3.0 & 23.3 & 3.8 & 26.7 & 6.0 & 26.4 & 6.7 & 23.5 & 5.1 & 18.6 & $<0.001$ & 0.01 \\
\hline
\end{tabular}

DMARD, disease-modifying antirheumatic drugs; RA, rheumatoid arthritis. 
Table 3 Multivariable analyses: methotrexate as the first DMARD versus hydroxychloroquine, and methotrexate as the first DMARD versus sulfasalazine

\begin{tabular}{|c|c|c|c|c|}
\hline & \multicolumn{4}{|c|}{ Choice of first DMARD } \\
\hline & \multicolumn{2}{|c|}{$\begin{array}{l}\text { Methotrexate versus } \\
\text { Hydroxychloroquine }\end{array}$} & \multicolumn{2}{|c|}{$\begin{array}{l}\text { Methotrexate versus } \\
\text { Sulfasalazine }\end{array}$} \\
\hline & $\overline{\text { OR }}$ & $95 \% \mathrm{Cl}$ & $\overline{\text { OR }}$ & $95 \% \mathrm{Cl}$ \\
\hline Time periods, 1999-2001 & 1.00 & & 1.00 & \\
\hline 2002-2003 & 1.07 & 0.89 to 1.29 & 1.06 & 0.82 to 1.37 \\
\hline 2004-2005 & $1.40^{\star *}$ & 1.17 to 1.68 & $1.53^{*}$ & 1.19 to 1.97 \\
\hline $2006-2007$ & $1.23^{\star}$ & 1.04 to 1.46 & $1.82^{\star \star}$ & 1.42 to 2.34 \\
\hline 2008-2009 & $1.57^{\star \star}$ & 1.28 to 1.93 & $2.10^{\star \star}$ & 1.56 to 2.85 \\
\hline \multicolumn{5}{|l|}{ Sex } \\
\hline Female & 1.00 & & 1.00 & \\
\hline Male & $1.58^{\star \star}$ & 1.31 to 1.91 & $0.72^{*}$ & 0.53 to 0.98 \\
\hline Ethnicity, Caucasian & 1.00 & & 1.00 & \\
\hline African American & 0.87 & 0.74 to 1.03 & 0.96 & 0.76 to 1.22 \\
\hline Others & 1.02 & 0.80 to 1.31 & $1.50^{*}$ & 1.01 to 2.24 \\
\hline Unknown/missing & 0.99 & 0.75 to 1.32 & 0.88 & 0.60 to 1.29 \\
\hline Age groups, $<45$ & 1.00 & & 1.00 & \\
\hline $45-54$ & 1.06 & 0.84 to 1.33 & 1.17 & 0.86 to 1.60 \\
\hline $55-64$ & $1.39^{\star}$ & 1.11 to 1.76 & $1.57^{\star *}$ & 1.14 to 2.16 \\
\hline $65-74$ & $1.57^{\star \star}$ & 1.23 to 2.02 & $2.18^{\star *}$ & 1.53 to 3.10 \\
\hline 75 or more & $1.59^{\star}$ & 1.21 to 2.08 & $3.70^{\star *}$ & 2.41 to 5.68 \\
\hline
\end{tabular}

five time periods (figure 1). Among the other independent variables used for adjustments, only age and ethnicity had significant associations with use of biologics. African Americans had 46\% chance of being dispensed biologics compared with Caucasians. Those aged 75 and older had $22 \%$ chance of being dispensed biologics compared with those younger than 45 (table 4 ).

At the end of our follow-up period, $59.3 \%$ of patients diagnosed with RA in 1999-2001 were still on their first DMARD or biological agent compared with $49 \%$ of those diagnosed in 2008-2009. The time between the

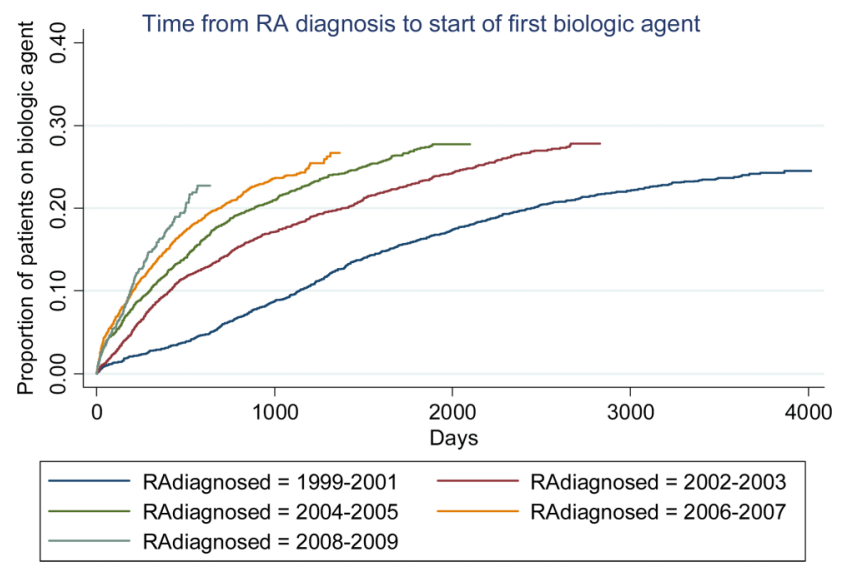

Figure 1 Kaplan-Meier plot of the proportion of patients placed on biological agents over time interval between rheumatoid arthritis diagnosis and the start of first biological agent (unadjusted results). first and the second DMARD or biological agent was shorter in recent years (median: 666 days (IQR 2509) in 1999-2001 to 256 days (IQR 318) in 2008-2009, MR 2.60 $(p<0.001))$. In the Cox regression model for therapeutic change, the HRs for therapeutic change were higher in the recent years. Those who were diagnosed with RA in 2008-2009 had 57\% higher chance of receiving a second DMARD or biological agent earlier than those diagnosed in 1999-2001. Patients aged 75 and older had $35 \%$ chance to have therapeutic change compared with those younger than 45 (table 4 ).

The trend of triple therapy usage did not change significantly over the five time periods $(\mathrm{p}=0.13)$. Over the 10 years of follow-up, only $327(2.5 \%)$ patients fulfilled our definition for triple therapy use.

\section{DISCUSSION}

Over the last decade, the management of RA has been transformed by an increasing number of treatment choices. The number of patients dispensed with MTX as their first DMARD or biological agent increased by $17.3 \%$ over the follow-up period, but the overall use of MTX increased by only $8.3 \%$. This was because of more frequent use of MTX as a second-line agent in earlier years and gradually increasing use of MTX as the firstline agent in later years. It was therefore not surprising to find a reciprocal decline in the percentage of patients on HCQ and SSZ. Leflunomide use as first-line DMARD did not change significantly over time, but its use as a second or subsequent agent trended lower over time, 
Table 4 Cox regression models for shorter time between $(A)$ rheumatoid arthritis diagnosis and the start of the first DMARD or biological agent, (B) rheumatoid arthritis diagnosis and start of the first biological agent and (C) start of the first and the start of the second DMARD or biological agent

\begin{tabular}{|c|c|c|c|c|c|c|}
\hline & \multicolumn{2}{|c|}{$\begin{array}{l}\text { Start of first DMARD or } \\
\text { biological agent }\end{array}$} & \multicolumn{2}{|c|}{$\begin{array}{l}\text { Start of first biological } \\
\text { agent }\end{array}$} & \multicolumn{2}{|c|}{$\begin{array}{l}\text { Start of second DMARD } \\
\text { or biological agent }\end{array}$} \\
\hline & $\overline{\text { HR }}$ & $95 \% \mathrm{Cl}$ & $\overline{\mathrm{HR}}$ & $95 \% \mathrm{Cl}$ & $\overline{\mathrm{HR}}$ & $95 \% \mathrm{Cl}$ \\
\hline Time periods, 1999-2001 & 1.00 & & 1.00 & 1.31 to 1.72 & 1.00 & \\
\hline 2002-2003 & 1.04 & 0.98 to 1.10 & $1.49^{\star *}$ & 1.66 to 2.17 & 1.05 & 0.98 to 1.12 \\
\hline 2004-2005 & $1.21^{\star *}$ & 1.15 to 1.28 & $1.90^{\star *}$ & 1.97 to 2.62 & $1.13^{\star \star}$ & 1.06 to 1.21 \\
\hline 2006-2007 & $1.33^{\star \star}$ & 1.26 to 1.40 & $2.27^{\star \star}$ & 2.32 to 3.37 & $1.22^{\star *}$ & 1.15 to 1.31 \\
\hline 2008-2009 & - & - & $2.80^{\star *}$ & & $1.33^{\star *}$ & 1.21 to 1.46 \\
\hline Ethnicity, Caucasian & 1.00 & & 1.00 & & 1.00 & \\
\hline African American & 0.95 & 0.90 to 1.01 & $0.71^{\star *}$ & 0.63 to 0.81 & 1.00 & 0.94 to 1.08 \\
\hline Others & 0.92 & 0.84 to 1.00 & 0.84 & 0.70 to 1.00 & 1.01 & 0.92 to 1.12 \\
\hline Unknown & 0.99 & 0.90 to 1.09 & 1.03 & 0.85 to 1.25 & 0.96 & 0.85 to 1.07 \\
\hline Age groups, $<45$ & 1.00 & & 1.00 & & 1.00 & \\
\hline $45-54$ & 1.08 & 0.99 to 1.17 & $0.84^{*}$ & 0.73 to 0.97 & 1.00 & 0.92 to 1.10 \\
\hline $55-64$ & $1.10^{*}$ & 1.01 to 1.19 & $0.73^{\star \star}$ & 0.64 to 0.85 & 0.93 & 0.84 to 1.01 \\
\hline $65-74$ & $1.20^{\star \star}$ & 1.10 to 1.31 & $0.48^{* *}$ & 0.41 to 0.57 & $0.67^{\star \star}$ & 0.61 to 0.74 \\
\hline 75 or more & $1.20^{* *}$ & 1.09 to 1.31 & $0.29^{\star *}$ & 0.23 to 0.36 & $0.53^{\star *}$ & 0.48 to 0.59 \\
\hline
\end{tabular}

dropping most significantly between the 2004-2005 and 2006-2007 periods. The higher likelihood of males using MTX as the first DMARD compared with HCQ may be explained by MTX being a class D drug for pregnancy, whereas HCQ is relatively safe during pregnancy. However, males were less likely to use SSZ as the first DMARD than MTX and this observation may be explained by SSZ possibly causing oligospermia.

Use of biologics as first-line DMARDs and biological agents increased over the years. However, the percentage of patients who had biologics dispensed remained stable through the first four time periods. There was clearly a pent-up demand for biologics among RA patients who were diagnosed in the earlier years, leading the significant rise in the actual number of biological agent dispensations across the five time periods. With the long-time interval between RA diagnosis and start of first biological agent, the percentage of patients eventually dispensed with biologics increased with time. The Kaplan-Meier curves in figure 1 showed that percentages of patients on biologics plateauing below $30 \%$ for those diagnosed with RA in 1999-2005, but the percentages of patients on biologics with RA diagnosed in 2007-2009 were still increasing at the end of the follow-up period. About 23.3-26.7\% of RA patients were on biologics. This overall percentage was lower than those reported in other databases. A study using the CORRONA database reported $1 / 3$ of RA patients on tumour necrosis factor inhibitors. ${ }^{7}$ The difference could be explained by its cohort having younger patients (mean age 59 compared with ours, which was 63) and a lower percentage of African Americans (4.5\% compared with ours, which was $13.1 \%)$. Another possible explanation was that the biologics were introduced at a slower pace in our study. Moreover, at the end of our study, the proportion of RA patients diagnosed in later years being dispensed biologics was still increasing.

Since biologics were added to the RA treatment armamentarium in the late 1990s, several studies reported its increased use over time. ${ }^{14} 15$ Our results were similar for the initial years following RA diagnosis. However, we also showed that the percentage of patients on biologics plateaued over time was reaching approximately 20-30\%. This figure may be unique to the VA population, and we can only assume that other populations may follow a similar pattern. Although biologics as first-line RA treatment increased over time, the actual percentage of use was low ranging from $3 \%$ to $6.7 \%$. This was likely a result of VA-specific recommendations, which require the use of formulary agents such as MTX for at least 3 months before biologics can be used. ${ }^{16}$

The time between RA diagnosis and the start of DMARD or biological agent treatment is a good indicator of the quality care for RA patients. ${ }^{17}$ Our data showed a higher chance of having shorter intervals between RA diagnosis and first DMARD or biological agent use in more recent time periods. Over the same time periods, there were increasing odds of shorter duration between starting dates of first and second DMARDs and biological agents, suggesting that VA rheumatologists took less time to identify the needs for therapeutic change. The use of triple therapy did not change significantly with time, and this could be attributed to the very few numbers of patients on triple therapy.

Our results showed that VA rheumatologists have gradually changed their practice behaviours in RA treatment 
over the past 10 years. This could indicate that the quality of care for RA patients is improving. There are other possible factors that could influence the rate of change in our clinical practice behaviours, such as recommendations by various organisations such as the American College of Rheumatology and the European League Against Rheumatism and the marketing strategies of the pharmaceuticals. ${ }^{17} 18$ Other factors, such as consumer health literacy and social media may add to the influence in the rate of such change.

Our study has the advantage of having a large incident population of RA patients with a long follow-up period. ${ }^{19}$ The VA pharmacy data are able to track the dose and duration of DMARDs and biological agents dispensed to each subject accurately without recall bias. Unlike drugs like aspirin, which patients may find cheaper to purchase from a non-VA pharmacy and thus would not be captured in VA pharmacy files, DMARDs and biological agents would not likely have this problem. Weaknesses in our study include the inability to exclude the effects from possible confounders, for example, disease severity. ${ }^{20}$ To reduce confounding effects from anaemia, liver dysfunction, renal insufficiency and other comorbidities, we adjusted our results with the various laboratory results and comorbidity scores. The inability to include certain important factors that may affect the decisions to prescribed DMARDs or biologics, such as alcohol consumption, social economic status and history of tuberculosis, was a limitation in our study. The VA population which is predominantly male and older in age compared with the general population limits our ability to extrapolate our results to the general population.

\section{Author affiliations}

${ }^{1}$ Michael E. DeBakey VA Medical Center Health Services Research and Development Center of Excellence, Houston, Texas, USA

${ }^{2}$ Baylor College of Medicine, Houston, Texas, USA

${ }^{3}$ School of Nursing, University of Houston-Victoria, Katy, Texas, USA

Acknowledgements We would like to acknowledge Hong-Jen Yu for his help with data analysis.

Contributors BN conceived the idea of the study and was responsible for the design of the study. BN was responsible for undertaking acquisition of the data, data analysis, produced the tables and graph. AC and MMK provided the input for the data analyses and contributed to the interpretation of the results. $B N$ was the lead writer. BN and AC critically revise successive drafts of the manuscript. All authors read and approved the final manuscript.

Funding The research reported here was supported by the Department of Veteran Affairs, Veterans Health Administration, Health Services Research and Development Service (VA.SCV.1010./000-00.B_N). This work was also partly supported by the Veterans Affairs Health Services Research and Development Service Houston Center of Excellence (HFP90-020).

Disclaimer The views expressed are those of the authors and do not necessarily represent those of the Department of Veterans Affairs, Baylor College of Medicine or the University of Houston Victoria.

Competing interests None
Ethics approval Baylor College of Medicine IRB, Houston, Texas, USA.

Provenance and peer review Not commissioned; externally peer reviewed.

Data sharing statement No additional data are available.

\section{REFERENCES}

1. Weinblatt ME, Coblyn JS, Fox DA, et al. Efficacy of low-dose methotrexate in rheumatoid arthritis. $N$ Engl $J$ Med 1985;312:818-22.

2. Aletaha $D$, Smolen JS. The rheumatoid arthritis patient in the clinic: comparing more than 1,300 consecutive DMARD courses. Rheumatology (Oxford) 2002;41:1367-74.

3. Kremer JM. Methotrexate update. Scand J Rheumato 1996;25:341-4.

4. Salliot $C$, van der Heijde D. Long-term safety of methotrexate monotherapy in patients with rheumatoid arthritis: a systematic literature research. Ann Rheum Dis 2009;68:1100-4.

5. Smolen JS, Aletaha D, Machold KP. Therapeutic strategies in early rheumatoid arthritis. Best Pract Res Clin Rheumatol 2005;19:163-77.

6. Curtis JR, Chen L, Harrold LR, et al. Physician preference motivates the use of anti-tumor necrosis factor therapy independent of clinical disease activity. Arthritis Care Res (Hoboken) 2010;62:101-7.

7. Lee SJ, Chang $\mathrm{H}$, Yazici $\mathrm{Y}$, et al. Utilization trends of tumor necrosis factor inhibitors among patients with rheumatoid arthritis in a United States observational cohort study. J Rheumatol 2009;36:1611-17.

8. Chu LH, Portugal C, Kawatkar AA, et al. Racial/ethnic differences in receiving biologic DMARD treatment among california medicaid rheumatoid arthritis patients. Arthritis Care Res (Hoboken) 2013;65:299-303.

9. $\mathrm{Ng} \mathrm{B}$, Aslam F, Petersen $\mathrm{NJ}$, et al. Identification of rheumatoid arthritis patients using an administrative database: a Veterans affairs study. Arthritis Care Res (Hoboken) 2012;64:1490-6.

10. Thomas SL, Edwards CJ, Smeeth L, et al. How accurate are diagnoses for rheumatoid arthritis and juvenile idiopathic arthritis in the general practice research database? Arthritis Rheum 2008;59:1314-21.

11. Charlson ME, Pompei $\mathrm{P}$, Ales $\mathrm{KL}$, et al. A new method of classifying prognostic comorbidity in longitudinal studies: development and validation. J Chronic Dis 1987;40:373-83.

12. Radner H, Smolen JS, Aletaha D. Comorbidity affects all domains of physical function and quality of life in patients with rheumatoid arthritis. Rheumatology (Oxford) 2011;50:381-8.

13. Deyo RA, Cherkin DC, Ciol MA. Adapting a clinical comorbidity index for use with ICD-9-CM administrative databases. J Clin Epidemiol 1992;45:613-19.

14. Grijalva $\mathrm{CG}$, Chung $\mathrm{CP}$, Stein $\mathrm{CM}$, et al. Changing patterns of medication use in patients with rheumatoid arthritis in a medicaid population. Rheumatology (Oxford) 2008;47:1061-4.

15. Soderlin MK, Lindroth $Y$, Jacobsson LT. Trends in medication and health-related quality of life in a population-based rheumatoid arthritis register in Malmo, Sweden. Rheumatology (Oxford) 2007;46:1355-8.

16. Sales M. National PBM drug criteria for use: leflunomide and biologic disease modifying anti-rheumatic drugs. 2006. http://www. pbm.va.gov/Clinical\%20Guidance/Criteria\%20For\%20Use/DMARDs $\% 20$ for\%20Rheumatoid\%20Arthritis\%20(Leflunomide $\% 20$ and $\%$ 20Biologic\%20Agents),\%20Criteria\%20for\%20Use.pdf (accessed 10 Jan 2008)

17. Smolen JS, Landewe R, Breedveld FC, et al. EULAR recommendations for the management of rheumatoid arthritis with synthetic and biological disease-modifying antirheumatic drugs. Ann Rheum Dis 2010;69:964-75.

18. Singh JA, Furst DE, Bharat A, et al. 2012 update of the 2008 American College of Rheumatology recommendations for the use of disease-modifying antirheumatic drugs and biologic agents in the treatment of rheumatoid arthritis. Arthritis Care Res (Hoboken) 2012;64:625-39.

19. Boyko EJ, Koepsell TD, Gaziano JM, et al. US Department of Veterans Affairs Medical Care System as a resource to epidemiologists. Am J Epidemiol 2000;151:307-14.

20. Hudson M, Suissa S. Avoiding common pitfalls in the analysis of observational studies of new treatments for rheumatoid arthritis. Arthritis Care Res (Hoboken) 2010;62:805-10. 